\title{
Natural Frequency Changes due to Severe Corrosion in Metallic Structures
}

\author{
Gilbert-Rainer Gillich* - Zeno Iosif Praisach - Vasile Iancu - Horia Furdui - Ionica Negru \\ "Eftimie Murgu" University of Resita, Faculty of Mechanical Engineering, Romania
}

Degradation of engineering structures due to corrosion affects their safety by reducing the cross-section of structural components and altering the material's mechanical characteristics. These parameter changes are observable in the shift of the natural frequencies. In the study presented in this paper, it was demonstrated that the kinetic energy distribution reflects the mass participation, thus being able to predict frequency changes due to mass loss, while the modal strain energy distribution can be properly used to indicate the location of the damage. As a result, two mathematical relations were developed by the authors, predicting the frequency changes due to the main effects of corrosion: loss of mass and loss of stiffness.

Keywords: corrosion, damage assessment, natural frequency, cantilever beam, mass loss

Highlights

- The effect of corrosion damage upon the natural frequency of structures is analyzed.

- Two mathematical relations for the prediction of frequency changes due to stiffness diminishing a respective loss of mass are proposed.

- The frequency increase due to loss of mass is proportional with the square of the mode shape at the damage location.

- The frequency drop due to stiffness decrease is proportional with the square of the mode shape curvature at the damage location.

- $\quad$ Simulations made using the ANSYS software and experiments confirmed the results achieved by calculus using the contrived relations.

\section{O INTRODUCTION}

The interaction between engineering structures and the environment usually have corrosion as a consequence, producing important changes in the materials mechanical and physical properties and the structures geometry [1] and [2]. This leads to damage that often result in impairment of the structures functions. The corrosion process always starts from the components surface, but sometimes penetrates deep into the material.

A conventional criterion to classify the corrosion phenomena concerns the appearance of the corroded area [3]. In this regard, two basic forms of corrosion are defined: (a) generalized corrosion, which can be either uniform, when the component surface is affected at the same rate on a large area or nonuniform corrosion, which is characterized by variation of the corrosion rate in different regions of the surface; (b) localized corrosion, which is restricted to compact areas.

Some typical damage geometries and their models are illustrated in Fig. 1. Examining this figure, we can conclude that for uniform corrosion, pitting, erosion and cavitation corrosion, a loss of mass and a cross-section reduction are present. Therefore, these types of damage are best modeled by beams or plates with a stepwise variable thickness [4] and [5]. In the case of stress and fatigue corrosion, the cross-section reduction is very local, and no loss of mass is present. Hence, the damage influence upon the beam rigidity can be modeled as a decrease in the longitudinal elasticity modulus E.

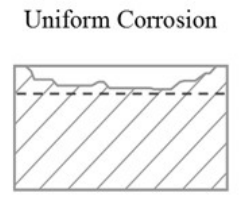

Cavitation

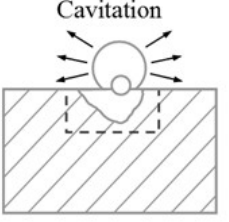

Fig. 1. Typical corrosion geometries and the equivalent geometrical models

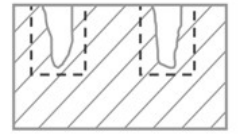

Erosion
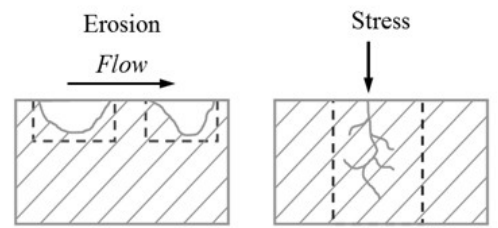

Herein, only corrosion causing both losses of mass and rigidity is considered. The thickness loss produced to structural elements by corrosion leads to a reduced bearing area and, therefore, decreased structural performance. This results in the lifeservice limitation, as shown in [6] and [7]. In addition, changes in the structure's modal parameters occur, 
mainly in the sense of natural frequency drops; these changes constitute the basic input if dynamic methods are applied to assess the damage. If cracks are present in the structure, the mass changes are insignificant and mainly neglected in most approaches.

In the case of generalized corrosion, it is impossible to neglect the loss of mass while this parameter becomes the most relevant one. Even if corrosion concerns the interest of researchers, because it is a major problem for structures, no reliable model for corroded structures is available.

In prior research, we considered the influence of transverse cracks, which did not produce loss of mass, upon the dynamic behavior of beams, and established a simple and reliable method to assess this type of damage [8]. The most recent investigations focused on the influence of both local and uniform corrosion on the beam dynamical behavior, with regard to the joined effect of stiffness change and loss of mass. This paper presents a behavioral model of corroded beams and the subsequent mathematical relations, as well as a procedure based on this model, used to improve damage detection methods.

\section{ANALYTICAL INVESTIGATION}

Since the natural frequency changes are the relevant features used for structural damage assessment, the aim of this section is to introduce two mathematical relations developed by the authors, indicating the changes due to stiffness decrease and loss of mass, respectively.

The case of a corroded cantilever beam is used for exemplification. Its geometrical asymmetry assures an unequivocal damage location definition. In its healthy state, the prismatic steel beam analyzed (Fig. 2) has the length $L$, the width $B$ and the height $H$. As a consequence, the beam has the cross-section area $A=B \cdot H$ and the moment of inertia $I=\left(B \cdot H^{3}\right) / 12$. The involved mechanical parameters are: the mass density $\rho$, the longitudinal elasticity modulus $E$ and the Poisson ratio $\mu$. In addition, the earth's gravity $g$ is considered. The single load acting on the cantilever is its own mass.

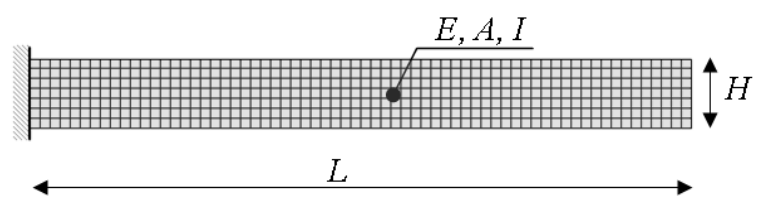

Fig. 2. Cantilever beam model
The natural frequencies $f_{i}$ for the bending vibration modes of a cantilever beam are derived as:

$$
f_{i}=\frac{\alpha_{i}^{2}}{2 \pi} \sqrt{\frac{E I}{m L^{3}}} .
$$

Here $m$ is the beam mass and $\alpha_{i}$ are the wave numbers for the beam that resulted as solutions of the characteristic vibration equation.

That is, for the cantilever beam:

$$
\cos \alpha \cosh \alpha+1=0 \text {. }
$$

In the case of uniform corrosion, the crosssection is reduced to $h$ on a segment of length $\Delta L$. The geometrical model is presented in Fig. 3a. To separate the effect of the stiffness reduction from that of the mass loss, two individual models are proposed. In the model depicted in Fig. 3b, the loss of mass is considered by reducing the volumetric mass density in the corroded region, while maintaining constant stiffness.

a)

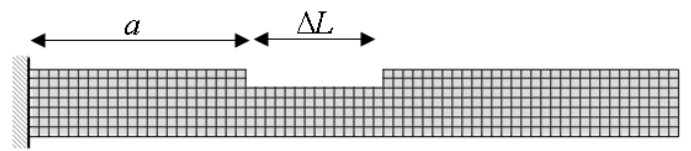

b)

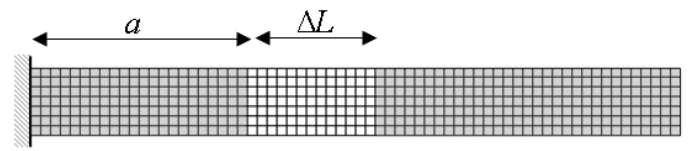

c)

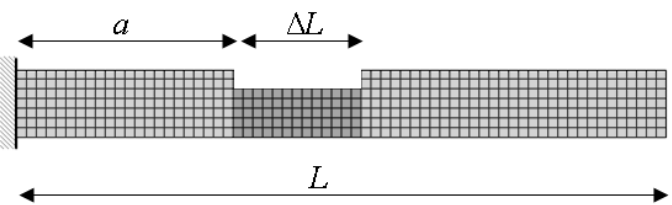

Fig. 3. Corroded cantilever beam models

Dissimilarly, to highlight just the effect of stiffness decreases, the model shown in Fig. $3 \mathrm{c}$ has an increased volumetric mass density in the corroded region.

The mass density is derived in both cases in such a way to assure the same beam mass.

\subsection{Deriving the Loss of Mass Influence}

The model presented in Fig. $3 \mathrm{~b}$ is used to derive the effect of the mass loss on the natural frequencies.

First, we consider the cantilever beam as continuous and having no mass. A mass $m_{P}$ located at the distance $x$ from the fixed end covers in a quarterperiod $T_{i} / 4$ the distance $w_{i}(x)$. A mass $m_{E}$ positioned at the free end cover in the same time distance wi $(L)$. 
These distances depend on the bending vibration mode shape, defined as:

$$
\begin{aligned}
& w_{i}(x)=\cos h \alpha_{i} x-\cos \alpha_{i} x- \\
& -\frac{\cos \alpha_{i} L+\cos h \alpha_{i} L}{\sin \alpha_{i} L+\sin h \alpha_{i} L}\left(\sin h \alpha_{i} x-\sin \alpha_{i} x\right),
\end{aligned}
$$

for the cantilever.

This fact is illustrated in Fig. 4 for the bending vibration mode three. The kinetic energy calculated from the mass $m_{P}$, the general case, is:

$$
U_{K i}(x)=\frac{m_{P}}{2}\left[\frac{w_{i}(x)}{T_{i}}\right]^{2} .
$$

For the mass $m_{E}$, the kinetic energy results in:

$$
U_{K i}(L)=\frac{m_{E}}{2}\left[\frac{w_{i}(L)}{T_{i}}\right]^{2} .
$$

To attain the same natural frequencies in both cases, the kinetic energies of the two models have to be equal. Eqs. (4) and (5) result in the dependency between two masses for the $i^{\text {th }}$ vibration mode, which is:

$$
m_{E i}=m_{P}\left[\frac{w_{i}(x)}{w_{i}(L)}\right]^{2}=m_{P}\left[\bar{w}_{i}(x)\right]^{2} .
$$

With $\bar{w}_{i}(x)$ we denoted the dimensionless transverse displacement for a point located at the distance $x$ from the fixed end; it takes values between null and the unit.
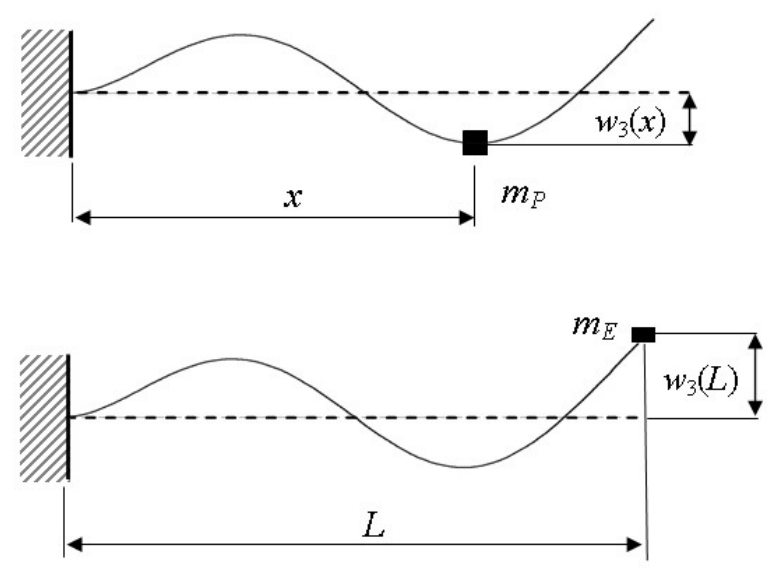

Fig. 4. Two dynamic equivalent systems, having the mass located at different positions

In concordance to Eq. (6), for any mass located at a distance $x$ from the fixed end, an equivalent mass conventionally located on the free end can be derived. These masses are in a relation given by the normalized mode shape square $\left[\bar{w}_{i}(x)\right]^{2}$. From the dynamic point of view, the beam equivalent own mass is achieved by summarizing the equivalent masses of all beam slices. Fig. 5 exemplifies the case of bending vibration Mode 3.
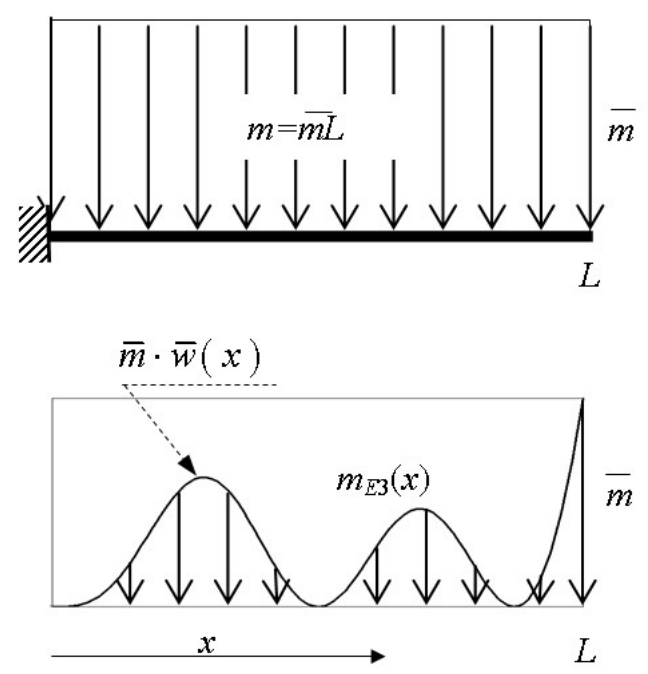

Fig. 5. Cantilever beam statically load by a distributed inertia and the mass participation in the kinetic energy for Mode three

In fact, the total equivalent mass $m_{i}^{e q}$, corresponding to the $i^{\text {th }}$ bending vibration mode, is obtained by multiplying the beam's specific mass $\bar{m}$ with the area under the curve $\left[\bar{w}_{i}(x)\right]^{2}$. Consequently,

$$
m_{i}^{e q}=\bar{m} L \int_{0}^{L}\left[\bar{w}_{i}(x)\right]^{2} d x=0.25 \bar{m} L .
$$

The area under the entire curve $\left[\bar{w}_{i}(x)\right]^{2}$ is 0.25 for all bending vibration modes, because the curves have the same nature.

Hence, the frequency of the undamaged beam can be expressed, with regard to the equivalent mass, as:

$$
f_{i}=\frac{\alpha_{i}^{2}}{2 \pi} \sqrt{\frac{E I}{\bar{m} L^{4}}}=\frac{\alpha_{i}^{2}}{2 \pi} \sqrt{\frac{E I}{4 m_{i}^{e q} L^{3}}} .
$$

The mass contribution to the total kinetic energy can be derived from Eq. (7) for any location along the beam. The participation of each slice regards the distance covered in a quarter of the period $T_{i}$, thus being different for the different vibration modes. Fig. 6 depicts the mass participation of 100 slices for the bending vibration mode three.

Let us consider now that the specific beam mass decreases due to uniform corrosion on a specific beam segment of length $\Delta L=b-a$. The consequence is a reduced specific mass $\bar{m}_{R}$ on that segment.

The equivalent mass contribution of the corroded segment is: 


$$
m_{R i}^{e q}=\bar{m}_{R} L \int_{a}^{b}\left[\bar{w}_{i}(x)\right]^{2} d x=\bar{m}_{R} L \varsigma_{i}
$$

Note that for corrosion extended on the whole beam surface, the mass participation coefficient is $\varsigma_{i}=0.25$.

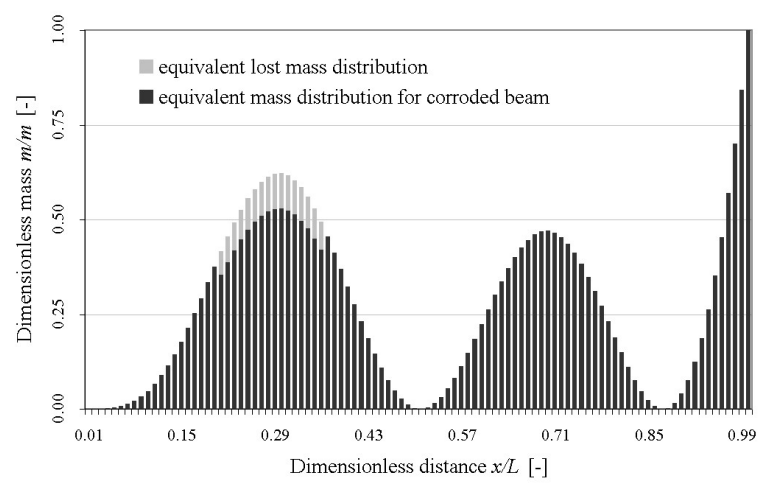

Fig. 6. The mass participation for 100 slices to the total kinetic energy for vibration Mode 3

Fig. 6 presents the mass participation for a beam having a corroded segment $(h=0.9 H)$, located between $a=0.2 L$ and $b=0.36 L$. On that portion, less mass will contribute to the kinetic energy. The entire mass contribution for the corroded beam is marked with dark bars in Fig. 6 .

The total equivalent specific mass, contributing to the kinetic energy, is found by summarizing the equivalent specific masses of the two healthy segments to that found for the corroded segment in Eq. (9). These are:

$$
\begin{aligned}
& m_{H i-1}^{e q}=\bar{m} L \int_{0}^{a}\left[\bar{w}_{i}(x)\right]^{2} d x=\bar{m} L \kappa_{i}, \\
& m_{H i-3}^{e q}=\bar{m} L \int_{b}^{L}\left[\bar{w}_{i}(x)\right]^{2} d x=\bar{m} L \sigma_{i},
\end{aligned}
$$

where $\kappa_{i}$ and $\sigma_{i}$ are the mass participation coefficient for the segments 0 to $a$ respectively $b$ to $L$.

We presumed the stiffness to be constant along the beam and the mass loss attributed to a decrease in the mass density, as illustrated in Fig. 3b. The natural frequencies for the beam with decreased mass are:

$$
f_{R i}=\frac{\alpha_{i}^{2}}{2 \pi} \sqrt{\frac{E I}{4\left(m_{H i-1}^{e q}+m_{R i}^{e q}+m_{H i-3}^{e q}\right) L^{3}}},
$$

similar to:

$$
f_{R i}=\frac{\alpha_{i}^{2}}{2 \pi} \sqrt{\frac{E I}{4\left(\kappa_{i} \bar{m}+\varsigma_{i} \bar{m}_{R}+\sigma_{i} \bar{m}\right) L^{4}}} .
$$

In regard to Eq. (8) and performing simple mathematical operations, the natural frequencies of the beam with the decreased mass result in:

$$
f_{R i}=f_{i} \sqrt{\frac{\bar{m}}{4\left(\kappa_{i} \bar{m}+\varsigma_{i} \bar{m}_{R}+\sigma_{i} \bar{m}\right)}} .
$$

The frequency increase caused by the loss of mass is:

$$
\Delta f_{R i}=f_{i}\left(\sqrt{\frac{\bar{m}}{4\left(\kappa_{i} \bar{m}+\zeta_{i} \bar{m}_{R}+\sigma_{i} \bar{m}\right)}}-1\right) .
$$

As a consequence, this has the relative frequency shift, attained by dividing the frequency increase to that of the healthy beam in the respective mode becomes:

$$
\Delta \tilde{f}_{R i}=\frac{\Delta f_{R i}}{f_{i}}=\sqrt{\frac{\bar{m}}{4\left(\kappa_{i} \bar{m}+\varsigma_{i} \bar{m}_{R}+\sigma_{i} \bar{m}\right)}}-1 .
$$

The frequency increases for two damage scenarios are illustrated below:

- in the first scenario (Fig. 7) the mass loss is simulated for segments of length $0.1 \mathrm{~L}$ and ten cases are analyzed, the location being successively replaced along the beam;

- in the second scenario (Fig. 8) the mass loss is simulated for segments of length $0.05 \mathrm{~L}$ and twenty cases are analyzed, the location being again successively replaced along the beam.

From the two figures presented below, one can first observe that the frequency shift due to loss of mass depends on the corroded segment's position on the beam. These changes are directly related to the mass participation (or the square of the highest velocity achieved by each slice individually), which is presented in Fig. 6. This is particularly obvious in Fig. 8 and confirmed by the expression of the kinetic energy, containing the sum of all beam slice masses.

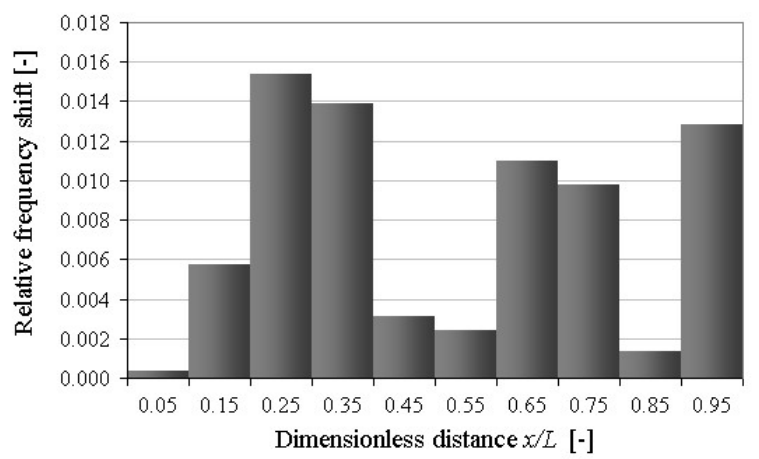

Fig. 7. The relative frequency shift for Mode 3: mass loss on segments of length $0.1 \mathrm{~L}$ 


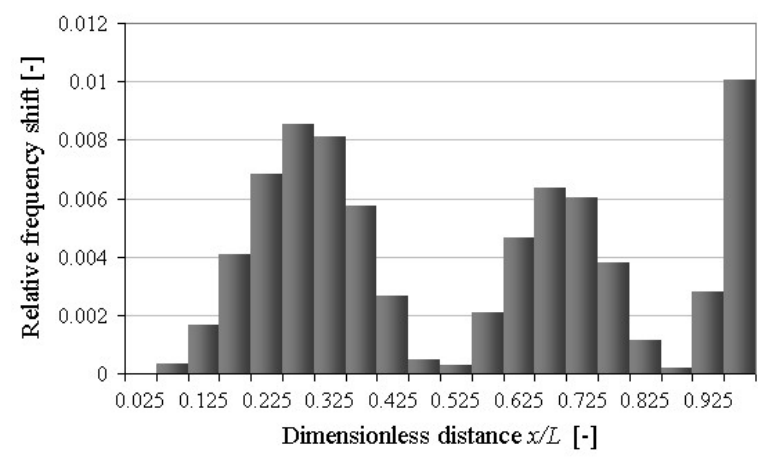

Fig. 8. The relative frequency shift for Mode 3: mass loss on segments of length $0.05 \mathrm{~L}$

Now, comparing the proportions between the bins in Fig. 7 and that of the homonym pair of bins in Fig. 8 a similarity is found, confirming the fact that the superposition principle is valid for the frequency shifts. This also can be deduced from Eq. (9).

\subsection{Deriving the Loss of Stiffness Influence}

The analysis made on the corroded beam by neglecting the mass decrease, according to the model presented in Fig. 3c, concerns only the moment of inertia $I$ as a factor influencing the natural frequencies. This case is nominated as corrosion in this sub-section.

In previous research [10], we proved that the energy loss is proportional to the deflection increase. The loss of capacity to store energy, due to damage to the corroded beam, manifests as an apparent energy increase for a healthy beam (the opposite to Castgliano's theorem), because of the deflection under similar load increases in case of damage.

Three specific domains can be observed in Fig. 9: the two healthy segments having the moment of inertia $I=B H^{3} / 12$, and the corroded segment with $I_{C}=B h^{3} / 12$.

Because we assume that the corroded segment has a higher mass density, the inertial forces will keep constant along the beam in order to compensate the mass loss. In the corroded region, the neutral axis is deviated to the new mid-cross-section.

While the mass distribution remains constant, the bending moment $M(x)$ is easily derived for the healthy cantilever beam, as it is known that it is proportional with the mode shapes' second derivative. Thus, the dimensionless bending moment is similar to the dimensionless curvature $\bar{M}_{i}(x)=\bar{w}_{i}^{\prime \prime}(x)$. Here, both terms of the relation are made dimensionless by the division to the highest value of the category, achieved at the fixed end: these are $M_{i}(0)$ and $w_{i}^{\prime \prime}(0)$.
In the case of a partial stiffness decrease, as shown in Fig. 9, it is convenient to consider the effective bending moment $M_{\text {eff }}(x)$. It is the moment acting on a presumed healthy beam, but producing a similar effect as $M(x)$ on the corroded one. The relation between the two bending moments is:

$$
M_{\text {eff }}(x)=M(x) \frac{I_{\text {actual }}}{I_{\text {reference }}} .
$$

As a consequence, this has a perturbation of the bending moment in the transition zones and an increase of it in the corroded region.
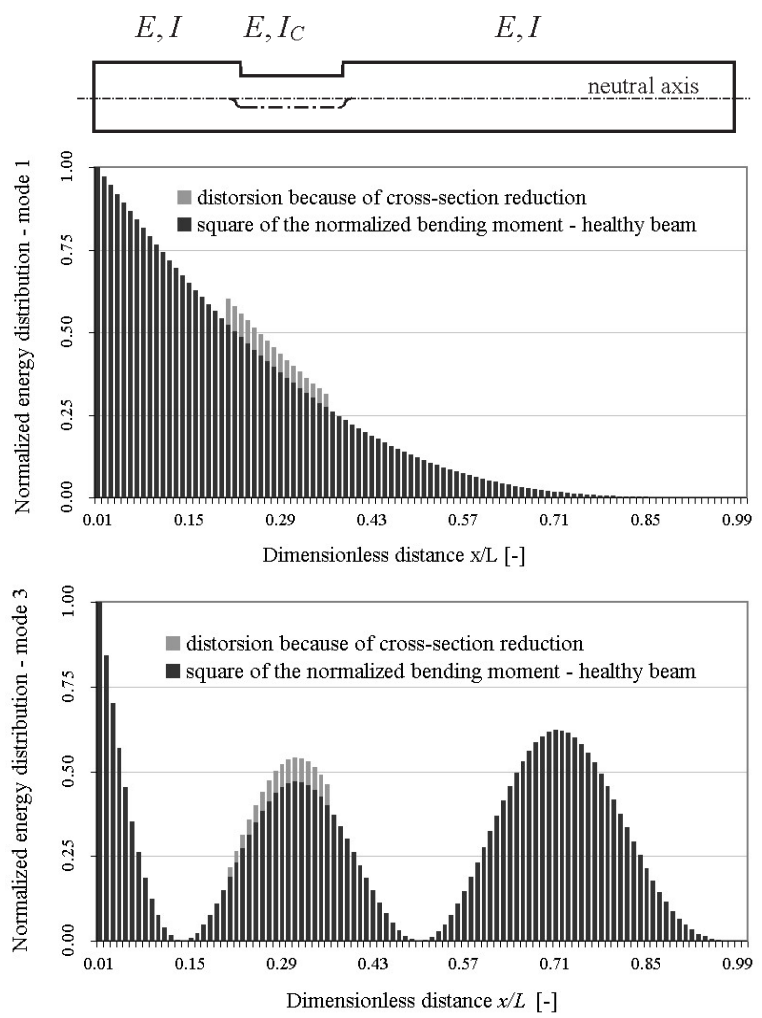

Fig. 9. Typical regions for a corroded beam and the energy distributions for modes 1 and 3

Furthermore, in Fig. 9, the apparent energy increase is presented for mode one (similar to the static load) and mode three. The distortion in the transition region can be neglected since the crosssection centers for the healthy and corroded segments are closely located.

The apparent energy increase is actually a decrease, while the beam is able to store less energy for a reduced cross-section. Thus, the energy loss ratio is derived from the corroded and healthy state, as: 


$$
\eta_{i}=\frac{\bar{U}_{S i}}{\bar{U}_{i}}=\frac{\frac{1}{2 E I} \int_{0}^{L}\left[\bar{M}_{e f f-i}(x)\right]^{2} d x}{\frac{1}{2 E I} \int_{0}^{L}\left[\bar{M}_{i}(x)\right]^{2} d x} .
$$

Both the actual and the effective moments are dimensionless.

The energy distribution along the beam for the analyzed case is, in the explicit form:

$$
\begin{aligned}
\bar{U}_{S i} & =\frac{1}{2 E I} \int_{0}^{a}\left[\bar{M}_{i}(x)\right]^{2} d x+ \\
& +\frac{1}{2 E I_{C}} \int_{a}^{b}\left[\bar{M}_{i}(x)\right]^{2} d x+\frac{1}{2 E I} \int_{b}^{L}\left[\bar{M}_{i}(x)\right]^{2} d x,
\end{aligned}
$$

where 0 to $a$ and $b$ to $L$ are the healthy segments and $a$ to $b$ is the corroded one.

In accordance with Eq. (16), and considering the inverse effect of stiffness decrease to the stored energy, we can replace the actual bending moment in Eq. (17) by $M(x)=\left(I_{C} / I\right) M_{C}(x)$. Moreover, by adding and subtracting the strain energy of the healthy segment $a$ to $b$ in Eq. (18), one attains:

$$
\begin{aligned}
\bar{U}_{S i} & =\bar{U}_{i}+\frac{1}{2 E I_{C}} \int_{a}^{b} \frac{I_{C}^{2}}{I^{2}}\left[\bar{M}_{i}(x)\right]^{2} d x- \\
& -\frac{1}{2 E I} \int_{a}^{b}\left[\bar{M}_{i}(x)\right]^{2} d x,
\end{aligned}
$$

or, in a comprehensive form:

$$
\bar{U}_{S i}=\bar{U}_{i}-\left(1-\frac{I_{C}}{I}\right) \frac{1}{2 E I} \int_{a}^{b}\left[\bar{M}_{i}(x)\right]^{2} d x .
$$

Now, after substituting $U_{S i}$ in Eq. (17) and reduction of the common factor, the energy loss ratio becomes:

$$
\eta_{i}=\frac{\int_{0}^{L}\left[\bar{M}_{i}(x)\right]^{2} d x}{\int_{0}^{L}\left[\bar{M}_{i}(x)\right]^{2} d x}-\frac{I-I_{C}}{I} \frac{\int_{a}^{b}\left[\bar{M}_{i}(x)\right]^{2} d x}{\int_{0}^{L}\left[\bar{M}_{i}(x)\right]^{2} d x} .
$$

Denoting $\tau_{i}=\int_{a}^{b}\left[\bar{M}_{i}(x)\right]^{2} d x$, and knowing that $\int_{0}^{L}\left[\bar{M}_{i}(x)\right]^{2} d x=0.25$ as in the case of lateral deflection, the energy loss ratio is found as:

$$
\eta_{i}=1-\frac{4 \tau_{i}\left(I-I_{C}\right)}{I}
$$

From Eqs. (17) and (22), the relation between the natural frequencies for the beam with stiffness decrease and the healthy one results as:

$$
\frac{f_{S i}}{f_{i}}=\sqrt{\frac{U_{S i}}{U_{i}}}=\sqrt{\eta_{i}}=\sqrt{1-\frac{4 \tau_{i}\left(I-I_{C}\right)}{I}},
$$

hence, the frequencies for the damaged beam are:

$$
f_{S i}=f_{i} \frac{\sqrt{I-4 \tau_{i}\left(I-I_{C}\right)}}{\sqrt{I}} .
$$

The frequency shift due to stiffness decrease results from Eq. (24), as:

$$
\Delta f_{S i}=f_{i}-f_{S i}=f_{i}\left[1-\frac{\sqrt{I-4 \tau_{i}\left(I-I_{C}\right)}}{\sqrt{I}}\right],
$$

and as consequence the relative frequency shift is:

$$
\Delta \tilde{f}_{S i}=\frac{\Delta f_{S i}}{f i}=\frac{\sqrt{I}-\sqrt{I-4 \tau_{i}\left(I-I_{C}\right)}}{\sqrt{I}} .
$$

In the following, the frequency decrease is illustrated for two damage scenarios. First, the stiffness reduction manifests on a segment of length $0.1 \mathrm{~L}$, and ten cases are analyzed. The damage location is successively replaced along the beam (see Fig. 10).

The second scenario considers a stiffness reduction on a segment of length $0.05 \mathrm{~L}$ and twenty cases are analyzed, the location again being successively replaced along the beam. The results are presented in Fig. 11.

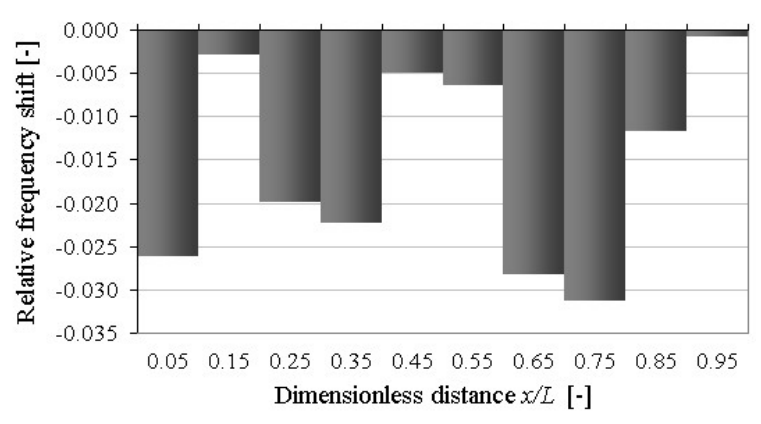

Fig. 10. The relative frequency shift for mode 3: stiffness loss on segments of length $0.1 \mathrm{~L}$

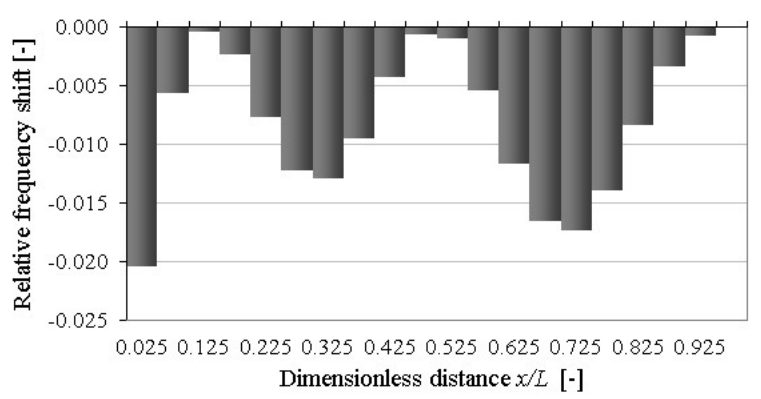

Fig. 11. The relative frequency shift for mode 3: stiffness loss on segments of length $0.05 \mathrm{~L}$ 
If the corrosion is extended on the whole beam surface, i.e. $\tau_{i}=0.25$, by neglecting the loss of mass, one obtains the frequencies for the slimmer beam (with thickness $h$ ) as:

$$
\hat{f}_{S i}=f_{i} \frac{\sqrt{I_{C}}}{\sqrt{I}} .
$$

The frequencies of a corroded beam (with both loss of mass and stiffness decrease) derived from Eqs. (16) and (24) depend on the healthy beam's frequency and 3 parameters that characterize the damage geometry: $\mathrm{a}$ and $\mathrm{b}$ indicating the damage extend and $d=H-h$ as the damage depth. Therefore, it is possible to characterize any corrosion damage by patterns, defined as a sequence of frequency shifts of some bending vibration modes. Comparing these patterns with frequency shifts found from measurements the damage can be located and its severity evaluated [9] to [11].

\section{NUMERICAL VERIFICATION}

In order to prove the validity of the contrived mathematical relations, which express the frequency changes due to mass and stiffness decrease, a finite element analysis was performed. First, the undamaged beam, as a reference, was investigated. Afterward, the corroded cantilever was considered, for three particular cases:

1. the beam with a corroded segment associated with loss of mass and stiffness, Fig. 3a;

2. the beam with constant stiffness having a segment presenting a loss of mass realized by the density alteration, Fig. 3b;

3. the beam having a segment with reduced stiffness, but with compensated loss of mass in order to assure the original mass, Fig. 3c.

The analysis was performed with SolidWorks software. Tetrahedral elements are taken, with the reference dimension of $6 \mathrm{~mm}$. The resulted number of nodes is around 16,000 and the number of elements around 7,800 in all cases, assuring high precision.

The intact steel beam with a rectangular cross section has the following geometry: length $L=1 \mathrm{~m}$, width $B=50 \mathrm{~mm}$ and height $H=5 \mathrm{~mm}$. The physical-mechanical parameters are: the mass density $\rho=7850 \mathrm{~kg} / \mathrm{m}^{3}$, the longitudinal elasticity modulus $E=2.0 \cdot 10^{11} \mathrm{~N} / \mathrm{m}^{2}$ and the Poisson ratio $\mu=0.3$. The Earth gravity acceleration is taken $g=9.806 \mathrm{~m} / \mathrm{s}^{2}$ and the mass of the beam results as being $m=1.9625 \mathrm{~kg}$.

The parameters of the corroded region for three conceived damage scenarios are presented comprehensively in Table 1.
Table 1. Damage geometrical parameters

\begin{tabular}{ccccc}
\hline Case & $\begin{array}{c}\text { Depth, } D \\
{[\mathrm{~mm}]}\end{array}$ & $\begin{array}{c}\text { Left limit, } a \\
{[\mathrm{~mm}]}\end{array}$ & $\begin{array}{c}\text { Right limit, } b \\
{[\mathrm{~mm}]}\end{array}$ & $\begin{array}{c}\text { Extent, } \Delta L \\
{[\mathrm{~mm}]}\end{array}$ \\
\hline A & 0.5 & 500 & 600 & 100 \\
\hline B & 1 & 500 & 600 & 100 \\
\hline C & 0.5 & 700 & 900 & 200 \\
\hline
\end{tabular}

The first three frequencies for the weak-axis bending vibration modes are found using the Modal analysis module. Table 2 presents the results derived from the contrived relations, for the healthy beam and the three damage scenarios, while the results obtained by simulations are presented in Table 3 . The fit between the results obtained analytically and by means of FEA confirm the validity of the relations.

Table 2. Natural frequencies - analytically

\begin{tabular}{|c|c|c|c|c|c|}
\hline $\begin{array}{c}\text { Mode } \\
\quad i\end{array}$ & Scenario & $\begin{array}{c}f_{i} \\
{[\mathrm{~Hz}]}\end{array}$ & $\begin{array}{l}f_{R i} \\
{[\mathrm{~Hz}]}\end{array}$ & $\begin{array}{c}f_{S i} \\
{[\mathrm{~Hz}]}\end{array}$ & $\begin{array}{l}f_{C i} \\
{[\mathrm{~Hz}]}\end{array}$ \\
\hline \multirow{3}{*}{1} & A & \multirow{3}{*}{4.077} & 4.0901 & 4.0589 & 4.072 \\
\hline & $B$ & & 4.1034 & 4.0444 & 4.0708 \\
\hline & $C$ & & 4.1668 & 4.0744 & 4.1642 \\
\hline \multirow{3}{*}{2} & $A$ & \multirow{3}{*}{25.550} & 25.779 & 24.840 & 25.069 \\
\hline & $B$ & & 26.015 & 24.257 & 24.722 \\
\hline & C & & 25.739 & 25.246 & 25.435 \\
\hline \multirow{3}{*}{3} & A & \multirow{3}{*}{71.539} & 71.652 & 71.138 & 71.251 \\
\hline & $B$ & & 71.766 & 70.815 & 71.042 \\
\hline & C & & 72.070 & 68.788 & 69.319 \\
\hline
\end{tabular}

The frequencies of the corroded beam from Table 2 are derived, involving the shifts due to the stiffness decrease and the mass loss, as:

$$
f_{C i}=f_{i}-\Delta f_{R i}-\Delta f_{S i}=f_{R i}+f_{S i}-f_{i}
$$

\begin{tabular}{|c|c|c|c|c|c|}
\hline $\begin{array}{c}\text { Mode } \\
\quad i\end{array}$ & Scenario & $\begin{array}{c}f_{i}^{F E M} \\
{[\mathrm{~Hz}]}\end{array}$ & $\begin{array}{c}f_{R i}^{F E M} \\
{[\mathrm{~Hz}]}\end{array}$ & $\begin{array}{c}f_{S i}^{F E M} \\
{[\mathrm{~Hz}]}\end{array}$ & $\begin{array}{c}f_{C i}^{F E M} \\
{[\mathrm{~Hz}]}\end{array}$ \\
\hline \multirow{3}{*}{1} & A & \multirow{3}{*}{4.092} & 4.1047 & 4.0669 & 4.08 \\
\hline & $B$ & & 4.1181 & 4.0289 & 4.054 \\
\hline & $C$ & & 4.1821 & 4.0885 & 4.178 \\
\hline \multirow{3}{*}{2} & A & \multirow{3}{*}{25.639} & 25.870 & 24.732 & 24.973 \\
\hline & $B$ & & 26.111 & 23.521 & 24.012 \\
\hline & C & & 25.708 & 25.225 & 25.314 \\
\hline \multirow{3}{*}{3} & $\mathrm{~A}$ & \multirow{3}{*}{71.792} & 71.902 & 71.280 & 71.416 \\
\hline & $B$ & & 72.019 & 70.605 & 70.879 \\
\hline & $C$ & & 72.335 & 68.329 & 68.941 \\
\hline
\end{tabular}

Table 3. Natural frequencies - FEM simulations

To have an overview on the results, the relative frequency shifts are derived and compared. Eqs. (15) and (26) are again involved for the cases of mass loss 
respectively stiffness decrease. If both changes occur, we use

$$
\Delta \tilde{f}_{C i}^{F E M}=\frac{f_{C i}^{F E M}-f_{i}^{F E M}}{f_{i}^{F E M}} 100 .
$$

Table 4 presents the results achieved from the analytical study and Table 5 those from the FEM analysis.

Table 4. Relative frequency shifts - analytical

\begin{tabular}{ccccc}
\hline $\begin{array}{c}\text { Mode } \\
i\end{array}$ & Scenario & $\Delta \tilde{f}_{R i}[\%]$ & $\Delta \tilde{f}_{S i}[\%]$ & $\Delta \tilde{f}_{C i}[\%]$ \\
\hline \multirow{3}{*}{1} & $\mathrm{~A}$ & 0.321 & -0.444 & -0.122 \\
\cline { 2 - 5 } & $\mathrm{B}$ & 0.647 & -0.799 & -0.152 \\
\cline { 2 - 5 } & $\mathrm{C}$ & 2.202 & -0.063 & 2.138 \\
\hline \multirow{3}{*}{2} & $\mathrm{~A}$ & 0.89 & -2.778 & -1.882 \\
\cline { 2 - 5 } & $\mathrm{B}$ & 1.819 & -5.060 & -3.240 \\
\cline { 2 - 5 } & $\mathrm{C}$ & 0.266 & -1.189 & -0.923 \\
\hline \multirow{3}{*}{3} & $\mathrm{~A}$ & 0.157 & -0.560 & -0.402 \\
\cline { 2 - 5 } & $\mathrm{B}$ & 0.317 & -1.012 & -0.694 \\
\cline { 2 - 5 } & $\mathrm{C}$ & 0.742 & -3.845 & -3.103 \\
\hline
\end{tabular}

Table 5. Relative frequency shifts - FEM analysis

\begin{tabular}{|c|c|c|c|c|}
\hline $\begin{array}{c}\text { Mode } \\
i\end{array}$ & Scenario & $\Delta \tilde{f}_{R i}^{F E M}$ & $\Delta \tilde{f}_{S i}^{F E M}$ & $\Delta \tilde{f}_{C i}^{F E M}[\%]$ \\
\hline \multirow{3}{*}{1} & A & 0.312 & -0.613 & -0.293 \\
\hline & $B$ & 0.637 & -1.540 & -0.928 \\
\hline & C & 2.203 & -0.085 & 2.101 \\
\hline \multirow{3}{*}{2} & A & 0.901 & -3.534 & -2.597 \\
\hline & $B$ & 1.843 & -8.258 & -6.345 \\
\hline & C & 0.269 & -1.613 & -1.349 \\
\hline \multirow{3}{*}{3} & A & 0.153 & -0.711 & -0.523 \\
\hline & $B$ & 0.317 & -1.652 & -1.271 \\
\hline & C & 0.756 & -4.823 & -3.971 \\
\hline
\end{tabular}

For a clear overview, the frequencies of the damaged beam are made dimensionless by dividing their values by the healthy beam's frequencies. The values, indicating a good concordance, are indicated in Fig. 12. A perfect fit is attained for the frequency increase due to mass loss derived by calculus and attained from the FEM. Regarding the case of stiffness decrease, the result varies, but with a value less than $3.5 \%$, which is achieved for Mode 2 of the beam with the deepest corrosion (the reason for this is the neglecting of the bending moment distortion). The correctness of the mathematical relations presented in Section 2 is confirmed.

The relative frequency shifts should be given a special attention. Their values are analogous for the two scenarios: A and B. The difference consists in their amplitudes, which are in a relation fixed by the damage severity (i.e. corrosion depth $d$ ). This shows that by normalizing the relative frequency shifts a sequence of values is achieved constituting a pattern that indicates the corroded region's location. Dissimilar is the case of Scenario C, which leads to a different pattern.

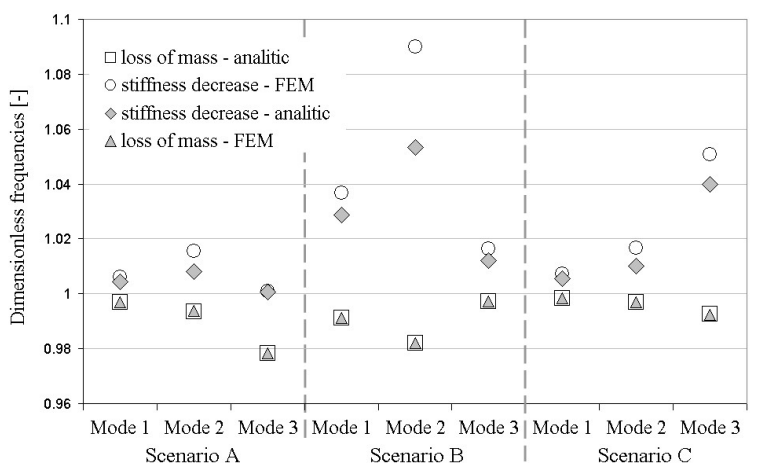

Fig. 12. Ratios between the frequencies derived analytically and evaluated by FEM analysis

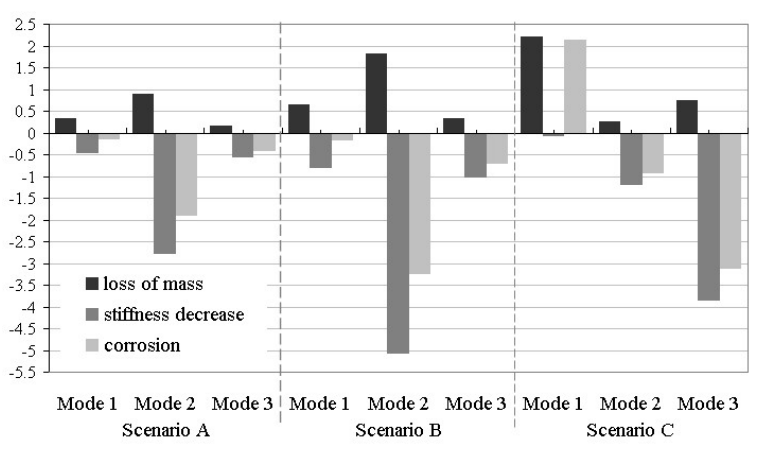

Fig. 13. The relative frequency shifts

Analyzing the frequencies of the beam in two damage locations, the results show that the position definitely determines the shift values. If a stiffness decrease affects an area near the contraflexure, less frequency drop is observed. In contrary, if the stiffness affects a region with important hogging, the drop is significant. The mass decrease leads to a frequency increase, which is in direct relation to the square of the mode shape value at the damage location.

\section{LABORATORY EXPERIMENTS}

The numerical analysis performed to prove the correctness of the theoretical findings was completed by experiments. These were performed on a similar cantilever, the three first natural frequencies for the healthy and corroded beam being aimed. To ensure proper rigidity for the clamped end, this was fixed in a milling machine vise, which is a component of the testing stand presented in Fig. 14. 
The acquisition equipment consists of a laptop computer, an NI cDAQ-9172 compact chassis with NI 9234 signal acquisition modules and a unidirectional Kistler 8772 accelerometer, which is fixed close to the beam's free end. High precision in evaluating the natural frequencies is required since a low shift in frequency is expected. For this reason, a virtual instrument was developed in LabVIEW that permits acquiring the acceleration time history and the accurate evaluation of frequencies [12].

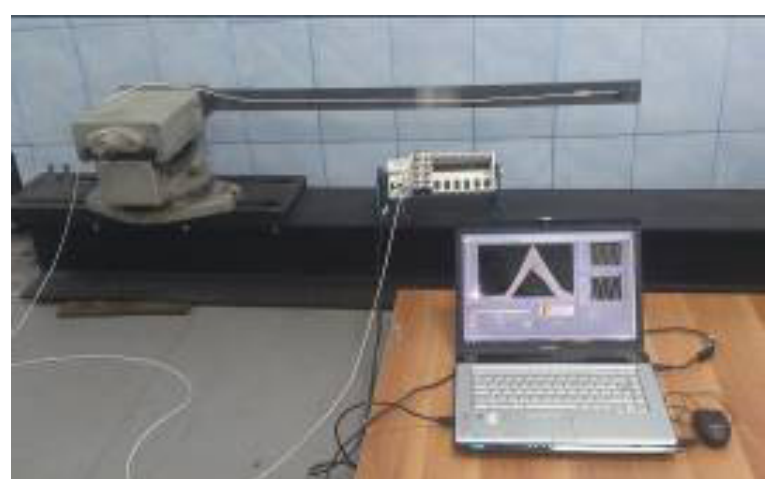

Fig. 14. The experimental stand

To simulate uniform corrosion, a discontinuity was produced by abrasion, the disruption having the parameter of scenario $\mathrm{A}$ in the numerical simulations section.

The measured frequencies $f_{i}^{M}$ for the first six bending vibration modes, for the intact beam and the beam with simulated corrosion, are presented in Table 6. Furthermore, the resulted relative frequency shifts are indicated, as well as the values derived by means of FEM analysis for the damage depth $d=0.5 \mathrm{~mm}$ respectively $d=1 \mathrm{~mm}$. The results, presented in Table 6 and Fig. 15, are in good correlation, confirming the validity of the proposed analytical relation for the prediction of frequency shifts.

Table 6. Measured natural frequencies and the relative shifts for the measured and calculated frequencies

\begin{tabular}{|c|c|c|c|c|c|}
\hline \multirow{2}{*}{$\begin{array}{c}\text { Mode } \\
i\end{array}$} & \multirow{2}{*}{$f_{i}^{M}[\mathrm{~Hz}]$} & \multirow{2}{*}{$f_{C i}^{M}[\mathrm{~Hz}]$} & \multirow{2}{*}{$\Delta \tilde{f}_{C i}^{M}[\%]$} & \multicolumn{2}{|c|}{$\Delta \tilde{f}_{C i}^{F E M}[\%]$} \\
\hline & & & & $d=0.5$ & $d=1$ \\
\hline 1 & 4.0199 & 3.9663 & -0.347 & -0.293 & -0.928 \\
\hline 2 & 25.192 & 23.535 & -5.881 & -2.597 & -6.345 \\
\hline 3 & 70.539 & 69.367 & -0.928 & -0.523 & -1.271 \\
\hline 4 & 138.23 & 132.54 & -3.402 & -1.506 & -3.374 \\
\hline 5 & 228.50 & 222.36 & -1.961 & -0.941 & -2.125 \\
\hline 6 & 341.34 & 332.46 & -1.875 & -0.990 & -2.235 \\
\hline
\end{tabular}

The frequency shifts derived by the FEA frame those from measurements, meaning the right location has been found. The damage depth, created between 0.5 and $1 \mathrm{~mm}$, is correctly estimated as $0.8 \mathrm{~mm}$. In contrast, by comparing the first three frequency shifts achieved by measurements with those in Table 5 for the case $\mathrm{C}$, dissimilarity can be observed. This proves the univocal definition of locations by the relative frequency shifts.

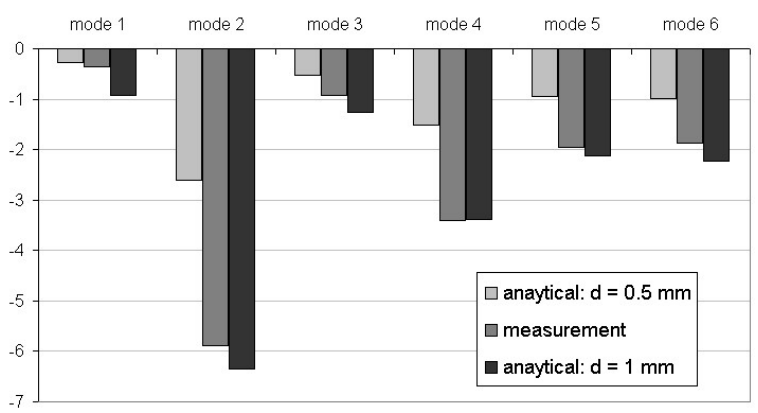

Fig. 15. Calculated relative frequency shifts for the FEM analysis and the measurement results

\section{CONCLUSION}

Two mathematical relations for the prediction of frequency changes due to stiffness diminishing a respective loss of mass are proposed. The first relation associates the effect of a local mass loss on the natural frequencies with the mode shape value corresponding to that location. The correlation between the frequency changes due to the stiffness decrease and the beam curvature was also found, and the second resulting relation was derived. It has to be mentioned that, due to the fact that in this second relation the effect of the bending moment distortion in the transition regions is neglected, deviations from the real frequency shifts can occur. This is a topic which is in our concern for feature research.

In order to reach the natural frequency values if both effects occur, the two relations have to be superimposed. Finite element analysis and experiments confirmed the correctness of these relations. Since the relations have as input the damage geometry and the healthy beams mode shape and curvature, these are easily adaptable for any boundary conditions.

The relative frequency shifts derived from these relations provide a simple way to describe the behavior of corroded beams. From these relations, a database containing numerous damage scenarios was realized. The corroded region in the three cases was successfully found with the involvement of this database. 


\section{ACKNOWLEDGEMENT}

The work has been funded by the Ministry of European Funds through the Financial Agreement POSDRU/159/1.5/S/132395.

\section{NOMENCLATURE}

A cross-section area of the healthy beam $\left[\mathrm{m}^{2}\right]$

$a$ distance from the fixed end to the left corrosion limit [m]

$B \quad$ beam width $[\mathrm{m}]$

$b$ distance from the fixed end to the right corrosion limit [m]

d corrosion depth [m]

$E \quad$ Young's modulus $\left[\mathrm{N} / \mathrm{m}^{2}\right]$

$f_{i} \quad i^{\text {th }}$ natural frequency $[\mathrm{Hz}]$

$\tilde{f}_{i} \quad$ relative frequency shift due to damage for the $i^{\text {th }}$ vibration mode [\%]

$H$ beam height [m]

$h$ height of the segment subjected to uniform corrosion [m]

I moment of inertia $\left[\mathrm{m}^{4}\right]$

$L$ beam length $[\mathrm{m}]$

$\Delta L \quad$ length of segment subjected to uniform corrosion [m]

$M(x)$ bending moment at distance $x$ from the fixed end $[\mathrm{N} \cdot \mathrm{m}]$

$M_{\text {eff }}(x)$ effective bending moment $x$ from the fixed end $[\mathrm{N} \cdot \mathrm{m}]$

$m_{P} \quad$ mass of a slice located at the distance $x$ from the fixed $[\mathrm{kg}]$

$m_{E} \quad$ mass of a slice located at the free end $[\mathrm{kg}]$

$m_{i}^{e q}$ equivalent mass of a beam segment for the $i^{\text {th }}$ vibration mode $[\mathrm{kg}]$

$\bar{m} \quad$ specific mass $[\mathrm{kg} / \mathrm{m}]$

$\bar{m}_{R} \quad$ reduced specific mass $[\mathrm{kg} / \mathrm{m}]$

$T$ period [s]

$w_{i}(x)$ beam deflection at distance $x$ from the fixed end for the $i^{\text {th }}$ vibration mode $[\mathrm{m}]$

$w_{i}^{\prime \prime}(x)$ beam curvature at distance $x$ from the fixed end for the $i^{\text {th }}$ vibration mode $\left[\mathrm{m}^{-1}\right]$

$U_{k} \quad$ kinetic energy $\left[\mathrm{kg} \cdot \mathrm{m}^{2} / \mathrm{s}^{2}\right]$

$\alpha_{i} \quad$ wave number of the $i^{\text {th }}$ vibration mode [-]

$\eta \quad$ energy loss ratio [-]

$\varsigma_{i}, \kappa_{i}$ and $\sigma_{i}$ mass participation coefficients of beam segments for the $i^{\text {th }}$ vibration mode [-]

$\rho \quad$ volumetric mass density $\left[\mathrm{kg} / \mathrm{m}^{3}\right]$

$\mu \quad$ Poisson ratio [-]

subscripts indicates

$i \quad$ the bending vibration mode number

$H \quad$ the healthy beam/segment parameters

$R$ the beam/segment parameters with reduced specific mass

$S \quad$ the beam/segment parameters with reduced specific stiffness

C the parameters of the corroded beam/segment superscript indicates

FEM the results obtained by means of the FEM

$M \quad$ the results obtained by measurements

\section{REFERENCES}

[1] ISO 8044 (1986). Corrosion of Metals and Alloys - Terms and Definitions. International Organization for Standardization, Geneva.

[2] Rahbar Ranji, A., Zakeri, A.H. (2010). Mechanical properties and corrosion resistance of normal strength and high strength steels in chloride solution. Journal of Naval Architecture and Marine Engineering, vol. 7, no. 2, p. 93-100, D0l:10.3329/ jname.v7i2.5309.

[3] Landolfo, R., Cascini, L., Portioli, P. (2010). modeling of metal structure corrosion damage: A state of the art report, Sustainability, vol. 2, no. 7, p. 2163-2175, Dol:10.3390/ su2072163.

[4] Bazant, Z.P. (1979). Physical model for steel corrosion in concrete sea structures-Theory. Journal of the Structural Division, vol. 105, no. 6, p. 1137-1153.

[5] Warkus, J., Raupach, M. (2010). Modelling of reinforcement corrosion - geometrical effects on macrocell corrosion. Materials and Corrosion, vol. 61, no. 6, p. 494-504, DOl:10.1002/mac0.200905437.

[6] Apostolopoulos, C.A., Michalopoulos, D. (2007). Impact of corrosion on mass loss, fatigue and hardness of BSt500 Steel. Journal of Materials Engineering and Performance, vol. 16, no. 1, p. 63-67, D0l:10.1007/s11665-006-9009-8.

[7] Zhang, X.Y., Li, S.-X., Liang, R., Akid, R. (2013). Effect of corrosion pits on fatigue life and crack initiation. 13th International Conference on Fracture, Beijing.

[8] Gillich, G.R., Praisach, Z.I., lavornic, C.M. (2012). Reliable method to detect and assess damages in beams based on frequency changes. Proceedings of the ASME International Design Engineering Technical Conference, Chicago, p. 129137, DOI:10.1115/DETC2012-70094.

[9] Thambiratnam, D., Shih, H.W., Chan, T., Tan, Z.X. (2015). Vibration based structural health monitoring to evaluate the damage in flexural members. International Journal of Mechanics, vol. 9, p. 181-188.

[10] Gillich, G.R., Praisach, Z.I. (2013). Damage-patterns-based method to locate discontinuities in beams. SPIE Proceedings, art. no. 869532, DOl:10.1117/12.2009122.

[11] Song, Y.Z., Bowen, C.R., Kim, H.A., Nassehi, A., Padget, J., Gathercole, N., Dent, A. (2014). Non-invasive damage detection in beams using marker extraction and wavelets. Mechanical Systems and Signal Processing, vol. 49, no. 1-2, p. 13-23, DOI:10.1016/j.ymssp.2013.12.011.

[12] Onchis-Moaca, D., Gillich, G.R., Frunza, R. (2012). Gradually improving the readability of the time-frequency spectra for natural frequency identification in cantilever beams. Proceedings of the 20 th European Signal Processing Conference, p. 809-813. 